\title{
New QCD Fits to HERA Data and Search for Exclusive Events at the Tevatron
}

\author{
Oldřich Kepka and Christophe Royon \\ DAPNIA/Service de physique des particules, \\ CEA/Saclay, 91191 Gif-sur-Yvette cedex, France
}

\begin{abstract}
We describe new QCD fits to diffractive proton structure functions measured at HERA, and we use these parton densities to predict the shape of the dijet mass fraction at the Tevatron and look for the existence of exclusive events in the dijet channel.
\end{abstract}

\section{QCD fits to proton diffractive structure function data from HERA}

We use the most recent published data [2] on diffractive proton structure function measured by the $\mathrm{H} 1$ and ZEUS collaborations. Data are fitted using the following quark and gluon densities [3]:

$$
\begin{aligned}
& z S\left(z, Q^{2}=Q_{0}^{2}\right)=\left[A_{S} z^{B_{S}}(1-z)^{C_{S}}\left(1+D_{S} z+E_{S} \sqrt{z}\right)\right] \cdot e^{\frac{0.01}{z-1}} \\
& z G\left(z, Q^{2}=Q_{0}^{2}\right)=\left[A_{G}(1-z)^{C_{G}}\right] \cdot e^{\frac{0.01}{z-1}} .
\end{aligned}
$$

In the fits, $\alpha_{S}\left(M_{Z}\right)=0.18$ and the initial scale is taken at $Q_{0}^{2}=3 \mathrm{GeV}^{2}$. The charm quark contribution is computed in the fixed flavour scheme using the photon-gluon fusion prescription. The pomeron intecept is found to be 0.12 using $\mathrm{H} 1$ data and $\chi^{2} /$ dof $\sim 0.9$. With respect to the "standard" H1 approach for the QCD fits, we have more parameters for the quark and gluon densities at the starting scale which allows to fix the starting scale at $3 \mathrm{GeV}^{2}$ and not to fit it. We cross checked that we find the same results as H1 while making the same assumptions. Other approaches based on dipole and saturation models [4] were also tested in Ref. [3].

The gluon and quark densities are given in Fig. 1. While the quark densities are found to be relatively close for $\mathrm{H} 1$ and ZEUS, the gluon density differs by more than a factor 2 . New preliminary data from ZEUS reduce this discrepancy. In the following, we will only use the QCD fits to the H1 data to compare with the dijet mass fractions measured in the CDF collaboration at the Tevatron. It is also worth noticing that the gluon density is poorly known at high $\beta$, where $\beta$ is the momentum fraction of the pomeron carried by the interacting parton. To illustrate this, we multiply the gluon density by the factor $(1-\beta)^{\nu}$ and fit the parameter $\nu$. The fit leads to $\nu=0.0 \pm 0.6$ which demonstrates a large uncertainty of the gluon density at high $\beta$ measured at HERA.

\section{Search for exclusive events at the Tevatron}

Exclusive events at the Tevatron or the LHC show the interesting property that the full available energy in the pomeron-pomeron system for double pomeron exchange events is used to produce the heavy mass object (dijet, diphoton...). In other words, no energy is lost in pomeron remnants. Tagging both protons scattered in the final state allow to measure precisely the kinematic properties, for instance the mass, of the produced heavy object. Exclusive events at the LHC recently captured high interest since it might be a possibility 

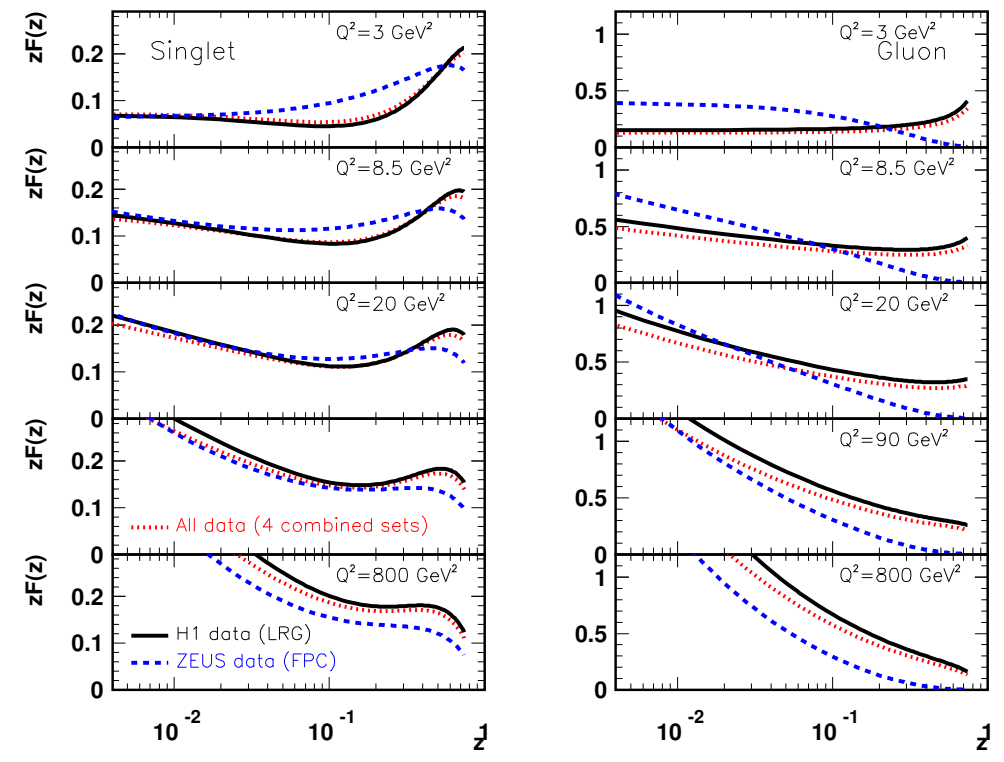

Figure 1: Gluon and quark densities in the pomeron measured using H1 and ZEUS data.

to detect the Higgs boson diffractively by tagging the diffracted protons in the final state [5].

\subsection{Search for exclusive events in $\chi_{C}$ production}

The CDF collaboration performed the search for exclusive events in the $\chi_{C}$ channel [6]. They obtained an upper limit of $\chi_{C}$ exclusive production in the $J / \Psi \gamma$ channel of $\sigma \sim 49 \mathrm{pb}$ $\pm 18 \pm 39 \mathrm{pb}$ for $y<0.6$. In Ref. [7], we found that the contamination of inclusive events into the signal region (the tail of the inclusive distribution when little energy is taken away by the pomeron remnants) depends stronly on the assumptions on the gluon distribution in the pomeron at high $\beta$ or in other words on the $\nu$ parameter. Therefore, this channel is unfortunately not conclusive concerning the existence of exclusive events.

\subsection{Search for exclusive events using the dijet mass fraction at the Tevatron}

One selects events with two jets only and one looks at the dijet mass fraction distribution, the ratio between the dijet mass and the total diffractive mass in the event. The CDF collaboration measured this quantity for different jet $p_{T}$ cuts [8]. We compare this measurement with different models of inclusive diffraction, namely "factorised" (FM) and "Bialas Landshoff" (BL) models [10]. In the FM models, one takes the gluon and quark densities in the pomeron measured at HERA as described in the previous section and the factorisation breaking between HERA and the Tevatron only comes through the gap survival probability. The BL model is non perturbative and diffraction is obtained via the exchange of a soft pomeron, which means that the mass dependence of the exclusive cross section is quite low. The comparison between the CDF data for a jet $p_{T}$ cut of $10 \mathrm{GeV}$ as an example and the predictions from the FM model is given in Fig. 2. We also give in the same figure the 


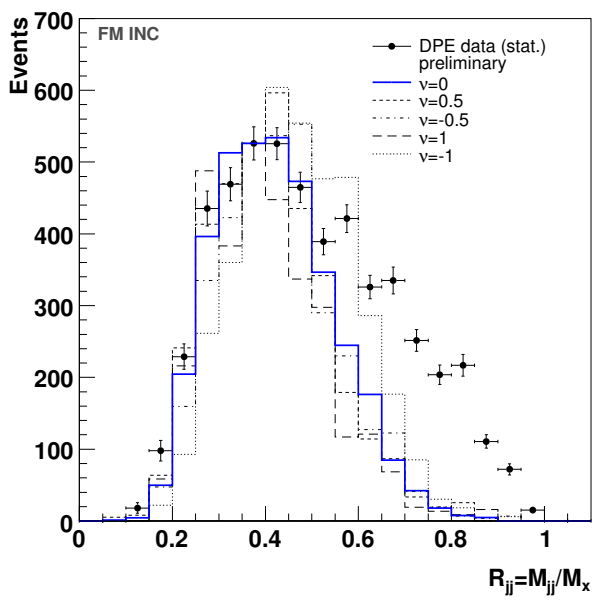

Figure 2: Dijet mass fraction measured by the CDF collaboration compared to the prediction from the "factorised model" for inclusive diffraction. The gluon density in the pomeron at high $\beta$ was modified by varying the parameter $\nu$.

effects of changing the gluon density at high $\beta$ (by changing the value of the $\nu$ parameter) and we note that inclusive diffraction is not able to describe the CDF data at high dijet mass fraction, where exclusive events are expected to appear [9]. The conclusion remains unchanged when jets with $p_{T}>25 \mathrm{GeV}$ are considered [9].

Adding exclusive events to the distribution of the dijet mass fraction leads to a good description of data [9] as shown in Fig. 3 where we superimpose the predictions from inclusive diffraction from the "factorised" model and exclusive one from the Durham model [10]. It is worth noticing that the exclusive "Bialas Landshoff" model [10] leads to a too small dependence of the diffractive exclusive cross section as a function of jet transverse momentum [9]. In Ref. [9], the CDF data were also compared to the soft colour interaction models [10]. While the need for exclusive events is less obvious for this model, especially at high jet $p_{T}$, the jet rapidity distribution measured by the CDF collaboration is badly reproduced. This is due to the fact that, in the SCI model, there is a large difference between requesting an intact proton in the final state and a rapidity gap [9].

\subsection{Observation of exclusive events at the LHC}

The exclusive contribution manifests itself as an increase in the tail of the dijet mass fraction distribution. Exclusive production slowly turns on with the increase of the jet $p_{T}$ (see Ref. [9]) and with respect to the uncertainty on the gluon density this appearance is almost negligible. The exclusive production at the LHC plays a minor role for low $p_{T}$ jets. Therefore, measurements e.g for $p_{T}<200 \mathrm{GeV}$ where the inclusive production is dominant could be used to constrain the gluon density in the pomeron. The higher $p_{T}$ jet region can be used to extract the exclusive contribution from the tail of the dijet mass fraction distribution. The extraction of the inclusive and exclusive jet production cross section will be of great importance at the beginning of the LHC to be able to make precise predictions on exclusive 


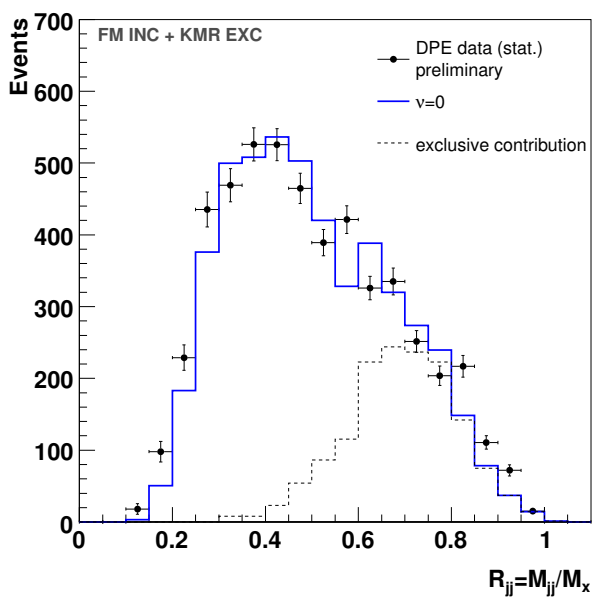

Figure 3: Dijet mass fraction measured by the CDF collaboration compared to the prediction from "factorised models" for inclusive diffraction and from the Durham model for exclusive diffraction.

Higgs production and the background later on.

\section{References}

[1] Slides: http://indico. cern. ch/contributionDisplay . py? contribId=242\&sessionId=7\&conf Id=9499

[2] A. Aktas et al. [H1 Collaboration], arXiv:hep-ex/0606004, arXiv:hep-ex/0606003; S. Chekanov et al. [ZEUS Collaboration], Nucl. Phys. B 713 (2005) 3.

[3] C. Royon, L. Schoeffel, R. Peschanski and E. Sauvan, Nucl. Phys. B 746 (2006) 15; C. Royon, L. Schoeffel, S. Sapeta, R. Peschanski and E. Sauvan, arXiv:hep-ph/0609291, accepted by Nucl. Phys. B.

[4] A.H.Mueller, Nucl. Phys. B415 (1994) 373; J.Bartels, J.Ellis, H.Kowalski, M.Wuesthoff, Eur.Phys.J.C7 (1999) 443; J.Bartels, C.Royon, Mod.Phys.Lett. A14 (1999) 1583; H.Navelet, R.Peschanski, Ch.Royon, S.Wallon, Phys. Lett. B385 (1996) 357; A.Bialas, R.Peschanski, C.Royon, Phys. Rev. D57 (1998) 6899; S.Munier, R.Peschanski, C.Royon, Nucl. Phys. B534 (1998) 297

[5] M. Boonekamp, R. Peschanski and C. Royon, Phys. Lett. B598 (2004) 243; M. Boonekamp, A. De Roeck, R. Peschanski and C. Royon, Phys. Lett. B 550 (2002) 93; M. Boonekamp, J. Cammin, S. Lavignac, R. Peschanski and C. Royon, Phys. Rev. D 73 (2006) 115011; V. A. Khoze, A. D. Martin and M. G. Ryskin, Eur. Phys. J. C23 (2002) 311, Eur. Phys. J. C24 (2002) 581.

[6] CDF Collaboration, T. Affolder at al., Phys. Rev. Lett. 85, 4215 (2000).

[7] M. Rangel, C. Royon, G. Alves, J. Barreto, R. Peschanski, hep-ph/0612297

[8] CDF Collaboration, CDF note 8493, (2006)

[9] O.Kepka, C. Royon, preprint arXiv:0704.1956

[10] M. Boonekamp, R. Peschanski, C. Royon, Phys. Rev. Lett. 87 (2001) 251806; M. Boonekamp, R Peschanski, C. Royon, Nucl. Phys. B669 (2003) 277; V. A. Khoze, A. D. Martin and M. G. Ryskin, Eur. Phys. J. C 48 (2006), Eur. Phys. J. C19 (2001) 477467; A. Bialas, P. V. Landshoff, Phys. Lett. B256 (1990) 540; R. Enberg, G. Ingelman, A. Kissavos, N. Timneanu, Phys. Rev. Lett. 89 (2002) 081801. 